
\section{$\varepsilon_{\mathrm{s}}$}

'Department of Radiology, Hospital Israelita Albert Einstein, Sao Paulo, Brazil ${ }^{2}$ Department of Thoracic Surgery, Hospital Israelita Albert Einstein, Sao Paulo, Brazil

Correspondence to Dr Gilberto Szarf, Department of Radiology, Hospital Israelita Albert Einstein, Av. Albert Einstein 627/701, Building D, 4th floor, Radiology Department, Sao Paulo, SP 05652-000, Brazil; gilbertos@einstein.br

Received 10 March 2015 Revised 13 June 2015 Accepted 25 June 2015 Published Online First 15 July 2015

\title{
Middle lobe pulmonary torsion after recurrent pleural effusions in a cirrhotic patient
}

\author{
Gilberto Szarf, ${ }^{1}$ Caroline Fontes R Dario, ${ }^{1}$ Rodrigo Caruso Chate, ${ }^{1}$ Elaine Yanata, ${ }^{1}$ \\ Eduardo Werebe, ${ }^{2}$ Marcelo Buarque Gusmao Funari ${ }^{1}$
}

A 57-year-old man was admitted with acute chest pain and shortness of breath. He had cirrhosis secondary to hepatitis $\mathrm{C}$ infection and had undergone multiple thoracocentesis in the last months for recurrent pleural effusions. Chest X-ray showed increased density in the right lung base and chest CT showed a right pleural effusion, partial atelectasis of the right lower lobe, middle lobe bronchovascular structures twisted on themselves with a swirling appearance, obliteration of the proximal middle lobe artery, narrowing of the accompanying bronchus and ground-glass opacities in this same lobe (figure 1). Symptoms persisted over the next week and a repeat chest CT again showed the swirl appearance of the bronchovascular structures of the middle lobe, more extensive ground-glass opacities,

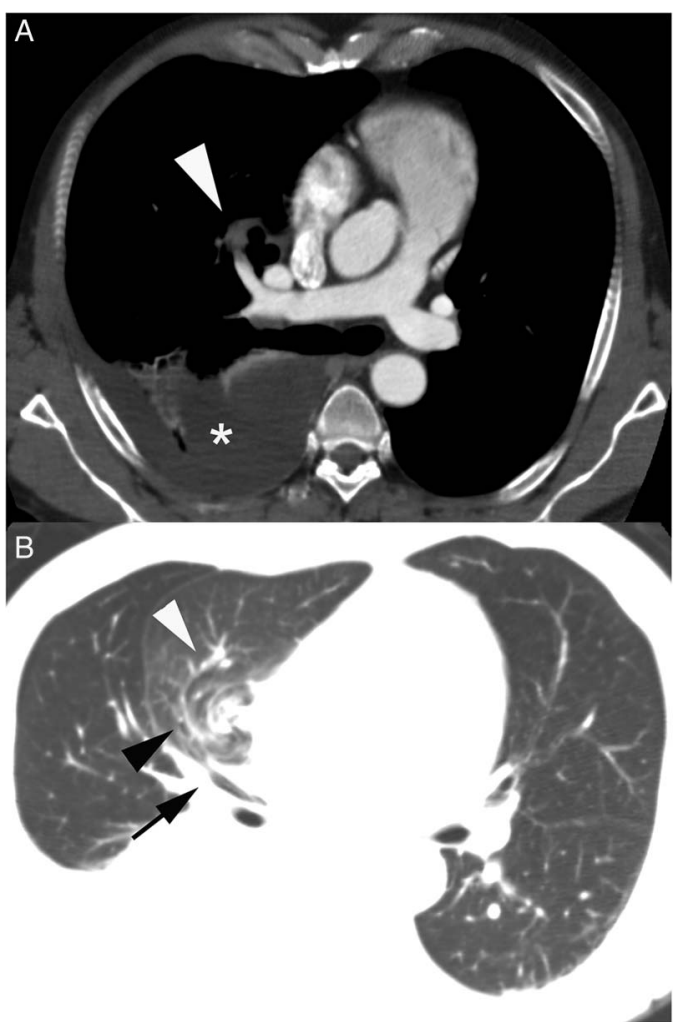

Figure 1 Chest CT reformatted image, showing (A) obliteration of the proximal middle lobe artery (white arrowhead), pleural effusion (asterisk) and partial right lower lobe atelectasis. (B) Narrowing of the accompanying bronchus (black arrow), middle lobe bronchovascular structures twisted on themselves with a swirling appearance (black arrowheads) and ground-glass pulmonary opacities (white arrowhead).
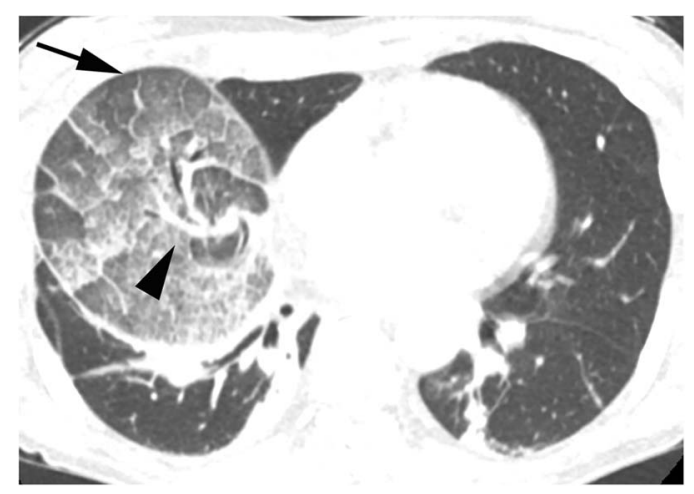

Figure 2 Chest CT obtained 7 days later, showing extensive ground-glass opacities and interlobular septal thickening (black arrow). The swirl appearance of the bronchovascular structures is again identified (black arrowhead).

areas of consolidation, interlobular septal thickening and increased middle lobe volume, probably as a result of venous congestion and infarction (figure 2). Open chest surgery confirmed middle lobe torsion, with signs of haemorrhagic infarction (figure 3). After detorsion, the lobe was non-viable, so resection was performed. Postoperative recovery was uneventful.

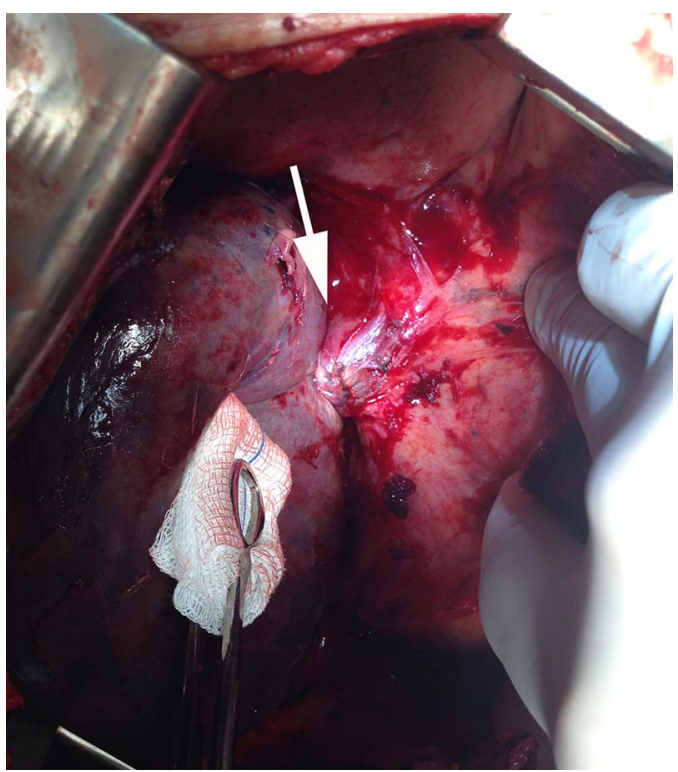

Figure 3 Intraoperative view of the right lung with torsion of the bronchovascular pedicle (white arrow). 


\section{Chest clinic}

Pleural effusions, as seen in this patient, can be a complication of cirrhotic patients. Passage of ascitic fluid through diaphragmatic defects, as a result of negative intrathoracic pressure and positive intra-abdominal pressure, seems to be the main cause.

Lung torsion is a very rare entity (reported incidence: 0.09\%$0.4 \%$ ), which is typically described as an adverse event after lung resection. ${ }^{1-3}$ It occurs when a lobe (or the complete lung) rotates around hilar structures. The degree of rotation is usually $180^{\circ}$, although in some instances $90^{\circ}$ or $360^{\circ}$ torsion can occur. ${ }^{4}$ Bronchovascular pedicle rotation can result in airway obstruction, venous compromise, ischaemia, infarction and gangrene. The clinical presentation of lung torsion is usually acute, but the rarity of this condition commonly results in a significant delay in diagnosis. Felson describes some radiographic signs of pulmonary torsion: collapsed or consolidated lobe, hilar displacement and unusual pulmonary vasculature positioning, rapid opacification of a pulmonary lobe, an opacified lobe positional change on sequentially obtained radiographs, bronchial cut-off or distortion and lobar air trapping. ${ }^{4}$ CT findings include tapered obliteration of the proximal pulmonary artery and accompanying bronchus of the involved lobe and amorphous soft-tissue attenuation at the hilum. The torsed lobe can show poorly enhancing consolidation with increased volume, ground-glass attenuation, interlobular septal thickening and intralobular linear attenuation.

Competing interests None declared.

Patient consent Obtained.

Provenance and peer review Not commissioned; externally peer reviewed.

\section{REFERENCES}

1 Wong PS, Goldstraw P. Pulmonary torsion: a questionnaire survey and a survey of the literature. Ann Thorac Surg 1992;54:286-8.

2 Cable DG, Deschamps C, Allen MS, et al. Lobar torsion after pulmonary resection: presentation and outcome. I Thorac Cardiovasc Surg 2001;122:1091-3.

3 Duan L, Chen X, Jiang G. Lobar torsion after video-assisted thoracoscopic lobectomy: 2 case reports. Thorac Cardiovasc Surg 2012;60:167-9.

4 Felson B. Lung torsion: radiographic findings in nine cases. Radiology 1987;162:631-8. 